NOTE

\title{
Validation of blood product irradiation doses
}

\author{
T Cheung ${ }^{1}$, M Butson ${ }^{1,2,3}$ and P K N Yu ${ }^{1}$ \\ ${ }^{1}$ City University of Hong Kong, Department of Physics and Materials Science, Kowloon Tong, \\ Hong Kong, People's Republic of China \\ 2 Illawarra Cancer Care Centre, Department of Medical Physics, Crown St, Wollongong, N.S.W \\ 2500, Australia \\ E-mail: mbutson@usa.net
}

Received 30 May 2001, in final form 10 July 2001

Published 20 September 2001

Online at stacks.iop.org/PMB/46/N241

\begin{abstract}
Dosimetry of blood irradiation using x-ray beams on a medical linear accelerator has been studied to evaluate the accuracy of a diode detector and the delivery achievable. Variations in applied doses for a standard dual field $6 \mathrm{MV}$ $\mathrm{x}$-ray are measured with a commercial diode detector. Results show that the diode detector measured applied in vitro doses to within 5.4\% (2 standard deviations (2 SD)) of those calculated with a collapsed-cone convolution treatment-planning computer for a sample of 100 blood irradiations. Experiments involving the packing procedure of the blood products in the blood box were performed. It was found that a large proportion of the variation in the predicted and measured dose was due to the compacting of the scatter material at the base of the blood box (over a 6 month period) producing a higher density below the blood than originally scanned; hence an overall reduction of delivered dose was observed. The diode measurements (which provide an immediate printout) are recommended in conjunction with a film dosimeter such as radiochromic film, which still provides a back-up dose monitor and a visual reminder that the blood has been irradiated. It is also recommended that the blood box be completely evacuated of all scatter material every month and the base be carefully repacked to provide uniform scatter material.
\end{abstract}

\section{Introduction}

Blood products are irradiated to diminish the risk of transfusion-associated graft versus host disease (TA-GVHD). The desired effect of irradiating the blood is to inhibit lymphocyte function and therefore to prevent GVHD while not causing damage to the platelets and other blood fractions. Guidelines based on the work of Pelszynski et al (1994) and Luban and

3 Author to whom correspondence should be addressed. 
DePalma (1996) state that at least 25 Gy provides a 5-log depletion of T-cells in a T-cell cologen assay which is sufficient for elimination of GVHD.

Various dosimetry techniques have been used to measure the dose to blood products. These include thermoluminescent dosimeters (TLDs); alanine, ferrous sulphate, red perspex, metal oxide semiconductor field effect transistors (MOSFETs) and chloroform/dithoizone/paraffin mixture (Hillyer et al 1993). Recently radiochromic film was shown to be an adequate dosimeter for blood irradiation (Butson et al 1999). The most prevalent method relies on TLDs. This work evaluates the use of a diode detector for immediate in vitro dose assessment and tries to ascertain the causes and variations in delivered in vitro dose across an 'active' treatment volume in a dedicated blood box for standard $\mathrm{x}$-ray beams.

\section{Materials and methods}

Blood products are irradiated in a $40 \mathrm{~cm} \times 40 \mathrm{~cm} \times 31.5 \mathrm{~cm}$ Perspex 'blood box' which is filled with the blood products to be irradiated and bags containing rice during irradiation. For standard blood irradiation, a dual parallel-opposed field $6 \mathrm{MV}$ treatment is used where the blood is placed in a $15 \mathrm{~cm}$ thick active volume, which is located in the centre of the blood box. Irradiations are performed using beams produced by a Varian $2100 \mathrm{C}$ medical linear accelerator. The blood is irradiated to an average dose of $30.6 \mathrm{~Gy}$. The minimum and maximum dose calculated within the active treatment volume is 29.6 and $31.7 \mathrm{~Gy}$, respectively. These values are calculated using a collapsed cone convolution algorithm from an ADAC pinnacle 3 planning system. In vitro measurements are made using a Scanditronix photon diode in absolute dose mode (Rikner 1985). This detector is attached to a dedicated dosimetry interface which measures integrated bias voltage on the diode. The collected reading is calibrated against measurements taken with the diode placed in standard conditions and irradiated to 30.6 Gy applied dose. That is, the diodes are placed in a water phantom and irradiated to $30.6 \mathrm{~Gy}$ applied dose at a depth of $10 \mathrm{~cm}$. The charge reading is compared to in vitro measurements. This standard calibration is performed at two weekly intervals to account for efficiency losses in the diode detectors.

During in vitro irradiations, the diodes are placed as close as possible to the beam's central axis at isocenter, which lies among the blood product bags being irradiated. One hundred in vitro irradiations and dose measurements were performed over a period of approximately 6 months using this technique. Due to variations in the measured dose, phantom measurements were performed using the diode detector to ascertain the sources of errors or deviations in measured/calculated doses. To perform this, the diode detectors were irradiated in the blood box, which was packed with four degrees of accuracy. (1) The rice bags in the bottom half of the blood box were packed once, then compacted 100 times over a 6 month period (this was the clinical situation as the bottom half of the blood box was never unpacked as it was unnecessary). (2) All the rice bags were carefully packed in and around the diode and the entire box to minimize any air gaps in the blood box phantom. (3) All the rice bags were placed in the blood box with some degree of accuracy but not packed into position to try and minimize any air gaps that could occur. (4) All the rice bags were thrown into the blood box in a disorganized manner. Each experiment was performed 20 times and the results for absolute dose and reproducibility were measured and compared to standard irradiation in the water phantom.

\section{Results and discussion}

Figure 1 shows a frequency histogram for the measured dose results in vitro for 100 blood irradiations using the $6 \mathrm{MV}$ x-ray dual field technique and the diode detector. The diode 


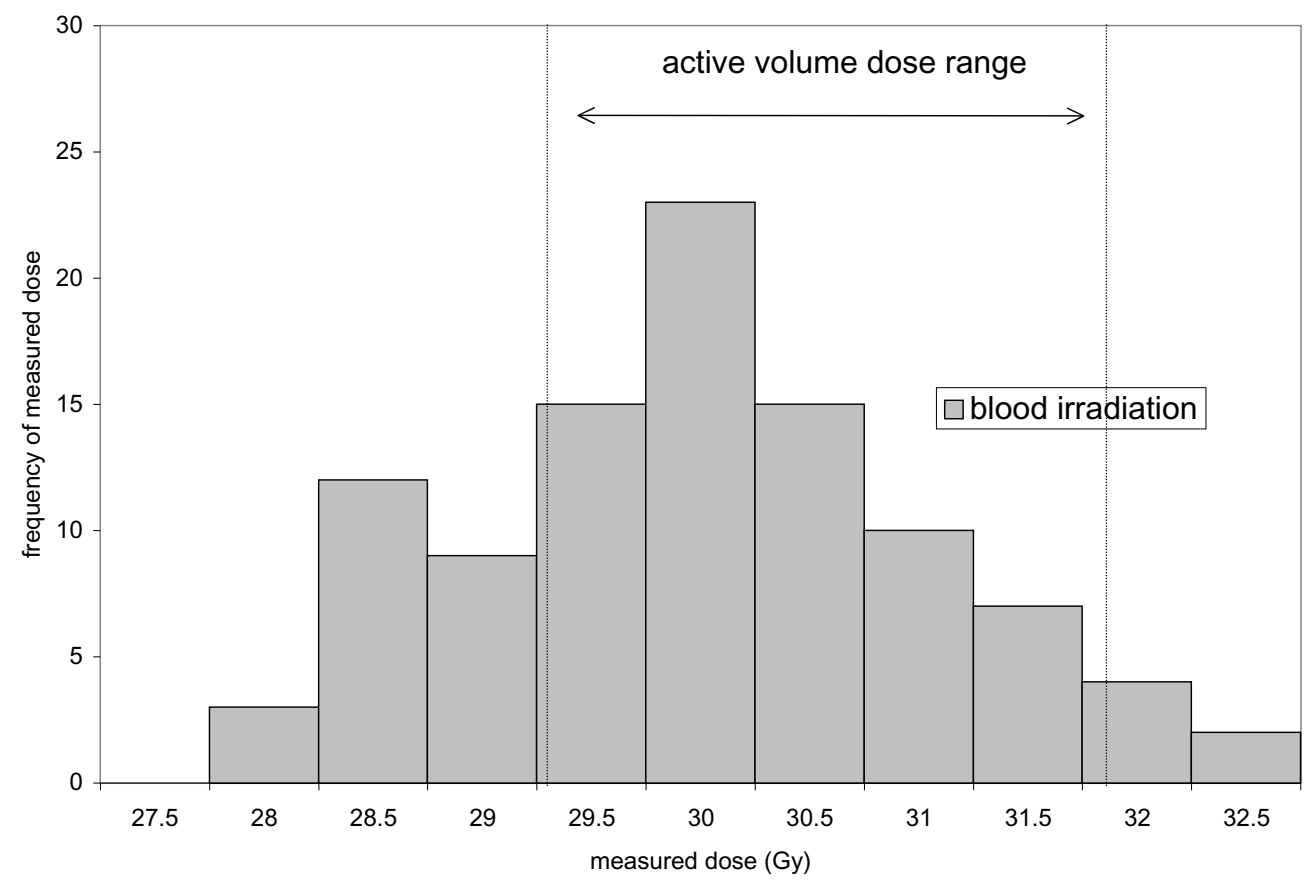

Figure 1. Results showing the histogram distribution of measured doses to blood products with 30.6 Gy applied dose using the diode dosimetry system.

detectors were placed next to the blood bags on each occasion. The mean dose measured was $30.2 \pm 1.6$ Gy for two standard deviations. Results show that the diode detector measures applied doses to within $5.4 \%$ (2 standard deviations (SD)) of the calculated doses in vitro for a sample of 100 blood irradiations. The diode detector provided an adequate measure of the in vitro dose. A variation of $5.4 \%$ in the measured dose may not be considered much when dealing with blood product irradiation. A minimum of $25 \mathrm{~Gy}$ is required when doses of the order of $30 \mathrm{~Gy}$ are given. This allows for up to $17 \%$ reduction in applied dose before the $25 \mathrm{~Gy}$ limit is reached. However, the results from the calibrations with diodes in water showed a less than $\pm 2 \%$ error in the diode-measured dose from day to day. Table 1 shows the results for the measured doses and errors obtained (2 SD of the mean) for the various configurations of packing mentioned. Results show a significant increase in errors as the randomness of packing increases. The absolute dose varied with the packing technique used. The dose delivered to the highly packed bags (1) was found to be on average $3.4 \%$ less than the carefully packed bags (2). This was assumed to be the main cause of the reduced average dose delivered in vitro for the blood products. As the base bags had been compacted over a 6 month period from initial CT and dose calculation, a higher density layer was developed. This reduced the delivered dose from the posterior field causing a slight reduction of the total delivered dose. This reduction was considered to probably be slightly offset by a more relaxed method of packing rice bags on top of the blood products (like experiment (3)) during in vitro irradiation. Based on these findings we would recommend to people using a similar blood box to completely evacuate the blood box of all scatter material every month and carefully repack the base to provide uniform scatter material as first performed before CT scanning. 
Table 1. Measured dose and variations with different packing techniques.

\begin{tabular}{lll}
\hline $\begin{array}{l}\text { Packing method } \\
\text { (Number) }\end{array}$ & $\begin{array}{l}\text { Average dose measured (Gy) } \\
(30.6 \text { Gy applied) }\end{array}$ & $\begin{array}{l}\text { Error } \\
(2 \mathrm{SD})\end{array}$ \\
\hline 1 & 29.6 & $\pm 0.8 \mathrm{~Gy}$ \\
2 & 30.4 & $\pm 1.1 \mathrm{~Gy}$ \\
3 & 30.9 & $\pm 1.5 \mathrm{~Gy}$ \\
4 & 31.5 & $\pm 2.0 \mathrm{~Gy}$ \\
\hline
\end{tabular}

\section{Conclusion}

Diode detectors used in absolute dose mode provide an accurate dosimetry verification tool for blood irradiation. They provide immediate dose. These detectors should be used in conjunction with a film dosimeter which provides a back-up dose monitor as well as a visual reminder that the blood has been irradiated. Variations in in vitro measured dose can be mainly attributed to changes in packing conditions and to the degree of compacting given to the scatter material around the blood products after CT scanning and dose calculation.

\section{Acknowledgments}

This work has been fully supported by a grant from the Research Grants Council of HKSAR, China (Project No. CityU 1137/00P).

\section{References}

Butson M, Yu P, Cheung T, Carolan M, Quach K, Arnold A and Metcalfe P 1999 Dosimetry of blood irradiation with radiochromic film Trans. Med. 9 205-8

Hillyer C D et al 1993 Development of a colorimetric dosimeter for quality control of blood units and irradiators Transfusion 33 898-901

Luban N L and DePalma L 1996 Transfusion-associated graft versus host disease in the neonate expanding the spectrum of disease Transfusion 36 101-3

Pelszynski M, Moroff G, Luban N, Taylor B and Quinones R 1994 Effect of gamma irradiation of red blood cell units on T-cell inactivation as assessed by limiting dilution analysis: Implications for preventing transfusion associated graft versus host disease Blood 83 1683-9

Rikner G 1985 Characteristics of selectivity shielded P-Si detector in Cobalt-60, 8 and 16 MV x-ray radiation Acta Radiol. Oncol. 24 205-8 